\title{
Perceptions of Rice Farmers towards Production Constraints: Case Study of Niger State of Nigeria and Hainan of China
}

\author{
Ahmed Abdul-Gafar ${ }^{1,2}$, Shiwei $X_{u^{1}}{ }^{*}$, Wen Yu${ }^{1}$ \\ ${ }^{1}$ Agricultural Information Institute, Graduate School of Chinese Academy of Agricultural Sciences, \\ Beijing, China \\ ${ }^{2}$ Partnership and Linkages, Agricultural Research Council of Nigeria (ARCN), Abuja, Nigeria \\ Email: abd.gafar1@icloud.com, xushiwei@caas.cn, yuwen@caas.cn
}

Received 7 March 2016; accepted 23 April 2016; published 30 April 2016

\begin{abstract}
Rice yield is still low in Nigeria despite the ecological advantages. Several challenges has been traced it production. The study intend to investigate if other rice producing nations are faced with similar challenges and at what magnitude and more importantly, what can be learn to improve the rice yield in Nigeria. Based on 2013/2014 survey, a total sample of 400 famers were randomly interviewed; 164 from Niger State of Nigeria and 236 from Hainan province of China. The study collate the perception of farmers to rice production constraints categorized into biotic, abiotic and socioeconomics. Biplot analysis was employed to examine multivariate pattern of their perceptions towards production constraints. The multivariate technique simultaneously displaying different yield levels and factor constraints in data matrix providing the inter-unit distances, variance and correlations of variables. According to the study, Niger state farmers identified socioeconomic constraint as the major factors to production and attributed it to lack of or insufficient investment while the Hainan farmers majorly identified abiotic constraints. The study also indicated that great potential remain to further improve rice yield in both regions especially in Nigeria given the appropriate investment on essential inputs. This study is of great use to extension officers more so, given the investment in Africa, policy makers take advantage of the bilateral and multilateral relationship to invest ease transfer of agricultural information and technologies between or among partners.
\end{abstract}

\section{Keywords}

Rice Farmers, Production Constraints, Biplot Analysis, Niger State, Hainan Province

\section{Introduction}

Global rice production is mostly concentrated in Asia as it's the primary staple food for most of the populace in the region, especially the rural area. With about $6 \%$ of global rice consumption, Africa accounts for about $4 \%$ of

\footnotetext{
${ }^{*}$ Corresponding author.
}

How to cite this paper: Abdul-Gafar, A., Xu, S.W. and Yu, W. (2016) Perceptions of Rice Farmers towards Production Constraints: Case Study of Niger State of Nigeria and Hainan of China. Journal of Agricultural Chemistry and Environment, 5, 20-30. http://dx.doi.org/10.4236/jacen.2016.51B004 
world production making the continent the second largest consuming and producing region. The increase in the demand for rice in Africa is majorly attributed to increase in income and population especially in West Africa. Unlike Asia, Africa's rice production is far less than the demand because of some production constraints. These constraints affect rice yield and it differs between countries depending on the seed variety, irrigation system, use of fertilizer and pesticides, water quality, climate and weather and in whole crop management (GRiSP 2013) [1]. Among the major producing countries, rice yield in Africa is still below world's average (4.3 MT), with more than half of West African countries having less than 2MT per hectare from 2008 to 2014. The Green Revolution has in a long way helped the Asian countries improve their rice productivity (USITC 2015) [2]. The quick adoption of several yield-improving technologies. Low productive countries are zealous to improve rice yield but majorly faced with improper crop management practices even with the presence of better seed variety. Lack of adequate extension services to assist rice farmers improve their crop management is one of the major barrier to improving productivity especially in West Africa. Another is as stipulated by Eponou (1993) [3] is that farming system from different regions are diverse and both agro-ecologically and sociologically challenged particularly in the developing countries. Hence, curbing the partnership and linkages problems relating to the difference in the nature of farming system by technology system for easing the transfer of agricultural technology and information is paramount.

This research intend to investigate and measure the production constraints faced by farmers and identify potential flexibility within which their farming systems can adopt changes from the view of two study areas. In order to fulfill these objectives, questions such as: what are the major factors that constrain rice yield? Ceteris paribus is the impact of these constraints largely/fairly different or similarly between the regions? And how are the natural, sociopolitical environment favoring the farmers? The goal here is to identify and provide potential strategies for partnering and linking agricultural technology and information, also providing a different and broader platform to extension offices on ways to combating production constraints.

\section{Study Area}

Niger State as the case study is chosen base on her ecological advantage of rice farming system (rain-fed upland and rain-fed lowland and irrigation) and natural water availability and as one of the leading rice producing states in Nigeria. Hainan province of China was purposely selected to be compared with Niger state on the bases of some common climate and major crops similarities shared between the two locations. Therefore, two-phase survey was conducted; one in Africa, Nigeria and the other in Asia, China.

\subsection{Niger State, Nigeria}

Location: Nigeria is located at the tropical zone of West African along the Atlantic Ocean's Gulf of Guinea lying between latitude 40 and $140^{\circ}$ north of the equator and longitude 30 and $140^{\circ}$ east of the Greenwich Meridian. Niger State is located at the Guinea Savanna vegetation zone in the north central part of Nigeria, lying between Latitude $8^{\circ} 22^{\prime} \mathrm{N}$ and $11^{\circ} 30^{\prime} \mathrm{N}$ and Longitude $3^{\circ} 30^{\prime} \mathrm{N}$ and $7^{\circ} 20^{\prime} \mathrm{E}$; and occupies land area of about $76,363 \mathrm{~km}^{2}$ of which $25 \%$ are water bodies. The state marks about $9 \%$ of the total land area in Nigeria, ranking the state as the largest in the country Figure 1(a) depicts the location and surveyed Local Government Areas.

Climate: Niger state experiences two seasons, the rainy (April to October) and dry season (November to March). The rainy season lasts for about 150 days in the Northern parts and about 120 days in the Southern parts of the State. The rains start at late April and ends in October with its peak in July and an annual rainfall ranging between 1100 to $1600 \mathrm{~mm}$ with mean relative humidity ranging between $60 \%$ in January to February and $80 \%$ between June to September. The average monthly maximum temperature $\left(35^{\circ} \mathrm{C}-360^{\circ} \mathrm{C}\right)$ is recorded between March and June, while the minimum $\left(20^{\circ} \mathrm{C}-210^{\circ} \mathrm{C}\right)$ is usually between December and January. (Niger state facts and figures, 2012).

Rice production takes place in all the ecological zones in Nigeria and comparatively the middle belt region has advantage over other regions. Niger state is located at the Middle-belt region and traditionally known as a rice growing area (Erenstein and Lancon 2003) [4]. Niger state contributes about 16\% of the national rice produced in the country. It is the second largest rice producing states in country after Kaduna state $19.63 \%$ (NBS, 2013) [5]. One of the major ecological problems hindering the rice sub-sector in the state is flooding during the rainy season, drought and bush burning during the dry season. Niger state rice yield estimate is about 2.3 metric ton per hectare although varies largely between farmers. 


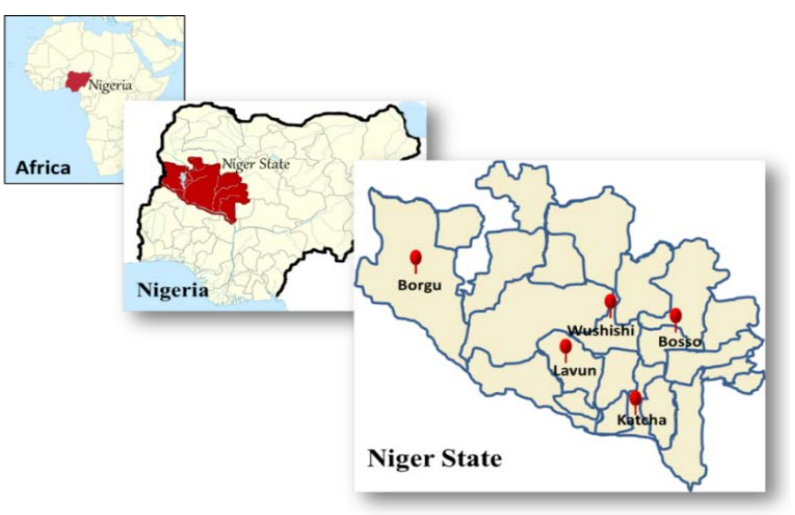

(a)

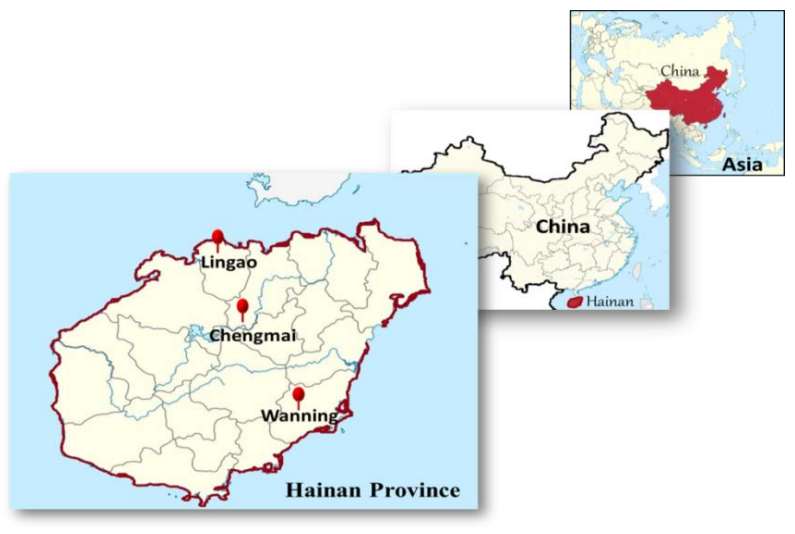

(b)

Figure 1. (a) Map-Niger state, Nigeria; (b) Map-Hainan, China.

\subsection{Hainan Province, China}

Location: Hainan province is an island located at the extreme south of China. It is located in $180^{\circ} 10^{\prime}-200^{\circ} 10^{\prime} \mathrm{N}$ and $1080^{\circ} 37^{\prime}-1110^{\circ} 03^{\prime} \mathrm{E}$ covering land area of about 35.4 thousand square kilometers and has maritime area of about 2 million square kilometers ranking the province as the 28th compare to other provinces in China. Figure 1(b) depicts the location of Hainan and the surveyed counties.

Climate: Hainan province belongs to tropical monsoon marine climate with a distinct dry and rainy/wet season. The dry season (during winter and spring) starts from January to May with average temperature $\left(22^{\circ} \mathrm{C}\right)$ during the day but colder during the night. The rainy season (during summer and autumn) begins from June and ends in November with an average temperature of $17^{\circ} \mathrm{C}$ at night and about $39^{\circ} \mathrm{C}$ during the day. The annual average temperature is $22.5^{\circ} \mathrm{C}-25.6^{\circ} \mathrm{C}$; the annual sunshine duration is 1780 - 2600 hours; the total solar radiation quantity is $4500-5800 \mathrm{MJ} / \mathrm{M} 2$. The annual rainfall is $1500-2500 \mathrm{~mm}$ (about $1000 \mathrm{~mm}$ for coastal areas in the west part). Major flooding occurs due to the typhoons and they can cause many problems for the local residents.

Rice production in Hainan province is commonly on landscapes. Rice is grown virtually in all the seasons and this makes the island a favorite site for seed companies for seed production. China's National Hybrid Rice R \& D Centre also has a station based near Sanya.

\section{Methodology}

\subsection{Selection Procedure and Sample Size}

Since the survey was based on rice production, the area where rice is produced was purposely selected and then a random selection procedure was carried out in each area. In Niger State five local governments were randomly selected on the basis of rice production and the share of land size. The local government areas include Borgu (western part of Niger state), Bosso, Katcha, Lavun and Wushishi local government. From a simple random se- 
lection, a total of 164 household were interviewed in the state. Two villages were selected in Borgu local government and one village from the other local government areas because of the large area and number of farmer in Borgu. In total six villages was covered. Moreover, in cases where applicable information is unavailable an alternative sources of secondary data such as National Bureau of Statistic (NBS), Food and Agricultural Organization (FAO), International Food Policy Research Institute (IFPRI), United State Department of Agriculture (USDA), Central Bank of Nigeria (CBN), World Bank, and Research Institutes and Government parastatals were employed.

In Hainan however, three counties were selected among which include Lingao (North-west of the province), Chengmai (Central), and Wanning (South-east). Three towns was selected from each county among which three villages were selected and lastly, a total of 236 farmers were randomly select and interviewed. Secondary data from multinational, national and provincial database were also used as an alternative source where applicable.

\subsection{Questionnaire Development}

The questionnaire was constructed in English, translated into Chinese in Agricultural Information Institute (AII) of Chinese Academy of Agricultural Science (CAAS), Beijing. The objectives of the survey are guided by the primary objectives of this study using the questionnaire. The questionnaire is divided into 5 parts: first part consist of questions on the socioeconomic status of the farmers such as the age, gender, years of education, household size; the second part consist of questions on production inputs such as the quantity/cost of fertilizer, labour, herbicide, pesticide and equipment/machines and seed variety; the third part consists of the output information such as quantity produced, quantity for food, seed or feed; fourth and fifth part include land utilization and production constraints.

\subsection{Data Processing and Method of Analysis}

Consequent upon the completion of the survey, the data input began in Agricultural Research Council of Nigeria (ARCN) and later completed in CAAS, China. EpiData software was use for data entry which afterward was transferred to STATA software for data generation and analysis. The unit to measure an area/field as well as the unit used for weighing agricultural products differs from country to country. For example in Nigeria, acre and hectares (ha) are the common units of measuring farm land while in China mu (i.e. $15 \mathrm{mu}$ equals to 1 hectare). In Nigeria the unit used for weighing produce is kilogram (kg), while in China grams (g) called "jin" in the local language (Mandarin). In this thesis all the units of measurement are converted into hectares and kilograms or tons.

There are several methods to analyzing perception, using simple statistical descriptive tools such as frequency and percentile (Oluwayomi D. A., 1984; Emechebe et al., 2004; Escalada et al., 2012) [6]-[8], multivariate analytical tools such as (Aerni and Yu 2010; Alarima et al., 2011) [9] [10]. In this study, both simple (tables) and multivariate (biplot) tools are used. Biplot (also referred to as a two-dimensional graph) is multivariate technique of simultaneously displaying observations (samples) and variables of a data matrix graphically; such information includes the inter-unit distances as well as variance and correlations of variables. The prefix "bi" refers to the simultaneous display of both rows and columns of the table, not to a two-dimensionality of the plot. Biplot was first described by Kuno Ruben Gabriel ${ }^{1}$ and recently extended by Gower and Hand (1996).Kohler and Luniak (2005) [11] [12] summarized the importance of biplot as revealing of clusters, multicollinearity, and multivariate outliers of a dataset, and could also be used to guiding the interpretation of Principal Component Analysis (PCA). In the data matrix, the observations are positioned on rows and the relative variable positioned on columns. Graphically on the other hand an example shown in Figure 2. The marker symbols (points) are displayed for observations and arrows are displayed for variables.

As mentioned above, the dots represent the observations and the arrow represents the variables. In the diagram above there are 12 observations and 7 variables. The length of the arrow approximates the variances of the variables; the longer the arrow the higher the variance and vice versa. The angle between the lines (cosine of the angles) approximates the correlation between the variables; the closer the angle is to 90 or 270 degree, the smaller the correlation. The correlation of 1 or -1 is reflected by an angle of 0 or 180 degree respectively. The distance between two points explains the Euclidean distance the two observations in the matrix space; observations that are closer to each other are then said to have low Euclidean distance and vice versa.

The Euclidean distance is defined as the square root of the sum of the squares of the differences between the corresponding coordinates of the points (dots). Giving an example of a two-dimension Euclidean geometry, the 


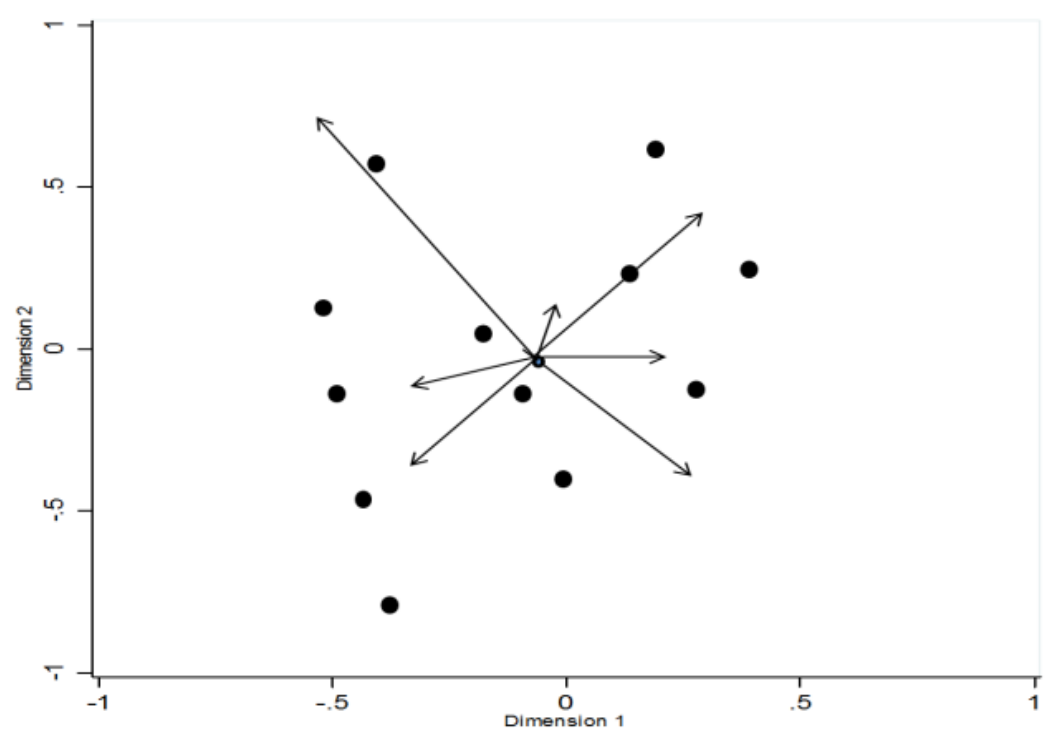

Figure 2. Biplot representing observations and variables.

Euclidean distance between two points say " $\mathrm{a}=\left(\mathrm{a}_{\mathrm{x}}, \mathrm{a}_{\mathrm{y}}\right)$ and $\mathrm{b}=\left(\mathrm{b}_{\mathrm{x}}, \mathrm{b}_{\mathrm{y}}\right)$ ” is defined as:

$$
d(a, b)=\sqrt{\left(\left(a_{x}-b_{x}\right)+\left(a_{y}-b_{y}\right)^{2}\right)}
$$

The ratio of the units of the vertical axis and those on the horizontal axis is also referred to as the aspect ratio, and is equal to 1.

\subsection{Mathematical Background of Biplot Analysis}

The Mathematical background of the biplot formula is summarized below. By using Singular Value Decomposition (SVD) ${ }^{1}$, the information in say matrix " $\mathrm{X}$ " is split into two; a portion related to the observations (i.e. the rows of $\mathrm{X}$ ) and another portion related to variables (i.e. the column of $\mathrm{X}$ matrix).

$$
\mathrm{X}=\mathrm{Y} \in \mathrm{Z}^{\prime}
$$

where $\mathrm{Y}$ is $n \times k, \in \& Z$ are $k \times \mathrm{k}$. the $\in$ (diagonal data) is known as the eigenvalues. From the SVD, the eigenvalue becomes:

$$
\in=\epsilon^{\alpha} \in^{1-\alpha}
$$

Therefore,

$$
\mathrm{X}=\mathrm{Y} \in^{\alpha} \in^{1-\alpha} \mathrm{Z}^{\prime}
$$

where $Y \epsilon^{\alpha}$ equal the coordinate for the observations and $\epsilon^{1-\alpha} Z$ equals the coordinate of the variables. Let denote $Y \epsilon^{\alpha}$ as "G" and $\epsilon^{1-\alpha} Z^{\prime}$ to be denoted as "H". The scalar Alfa ( $\alpha$ ) takes any value between zero and one (i.e. $0 \leq \alpha \leq 1$ ). Regardless of the value, the equation remains $\mathrm{GH}^{\prime}=\mathrm{Y} \in \in^{\alpha} \in^{1-\alpha} \mathrm{Z}^{\prime}=\mathrm{Y} \in \mathrm{Z}^{\prime}=\mathrm{X}$. However, as $\mathrm{G}$ is $n \times k$ and $\mathrm{H}$ is $k \times k$, all the coordinates have $k$ dimensions. To indicate the quality of the approximation of the information provided by the biplot, the default axis titles (dimension 1 and 2 i.e. $\mathrm{x}$ and $\mathrm{y}$ axis respectively) indicate the amount of explained variances by the selected dimensions.

\section{Result and Discussions}

\subsection{Farmers' Perception}

Farmers' perception can be of a great influence to farm management decision making just as the influence of

\footnotetext{
${ }^{1}$ See Johnson and Wichern (2007) [13] for more details on Single Value Decomposition (SVD).
} 
their economic situation to management decisions. The perception itself is influenced by education, years of experience, special training or extension programs. Several studies have been carried out on farmers' perception of constraints on agricultural production (Oluwayomi D. A., 1984; Emechebe et al., 2004; Sinzogan A.A.C et al., 2004; Ojehomon et al., 2009; Aerni and Yu 2010; Alarima et al., 2011; Atera et al, 2012; Escalada et al., 2012) [7]-[10] [14]-[17]. The perception of farmers has been one of the significant components used to evaluate technological adoption and it feedback, and management efficiency of farmers. Farmers' decision before, during and after the production process are constrained by both on-field and external factors. The comparison of production constraints of the farmers between two distant regions for the sole purpose of promoting agricultural technological and information transfer, the farmers interviewed were asked to rank constraints according to the severity. The constraints are categorized into biotic, abiotic and socioeconomic constraints. The result of how farmer perceive these constraint can be used to determine the knowledge and perception gaps amongst the famers.

\subsection{Categorization and Evaluation of Constraints}

Farmers were inquired to rank (from 0 to 3 ) each of the identified constraints according to the degree of occurrence/magnitude hindering production. Zero (0) indicates that such constraints doesn't exist or insignificant to affecting production decisions or output, one (1) indicates constraints as "low", two (2) and three (3) indicating "medium" and "high" respectively.

\subsubsection{Biotic Constraints}

The biotic constraints consist of constraints associated with living organisms such as plants and animals, this study summarizes the biotic constraints into three, namely weeds, bird/rodents and diseases/insects. In Hainan province, Diseases and pest infestation are considered as the highest biotic threat to rice production. With access and relatively low price of agrochemical, the higher the perceived threat of a farmer to certain constraint the more investment will be put into the input that reduces such threat. Low (2003) [18] and some other multinational organizations also highlighted the overuse of some agrochemicals among the Chinese farmers. In this study, over $50 \%$ of the farmers in Hainan perceived pest and diseases threat high while farmers in Niger state majority (about 43\%) believe the threat to be low.

\subsubsection{Aiotic Constraints}

Abiotic as a constraint are those factors that are not traceable to living organisms and majorly infers to the harsh weather and field ecological conditions such as flood, drought, erosion, heat stress and cold, and soil fertility. The abiotic factors were ranked according to the frequency of occurrence and/or damages. Majority (about 30\% in Niger state and 31\% in Hainan province) ranked the damage of flood to their crop as high. The average response to flood as constraint in both areas is ranked as the highest among the abiotic constraint factors listed above. Other factors (drought, soil fertility, soil erosion, heat stress and cold) showed clearer difference between Niger state and Hainan province. Comparatively, the average perception of Hainan farmers toward rice production constraint caused by abiotic factors is higher than that of farmers in Niger state (Figure 3(b)).

\subsubsection{Socioeconomic Constraints}

The socioeconomic constraint refers to the high cost and unavailability or difficulties to accessing essential factors of production such as credit, land, labour, seed, fertiliser, mechanisation while the indirect factors includes low market price, transportation cost and poor roads. The socioeconomic constraint also explains the reason why some farmers are unable apply or adopt the recommended management practice necessary to obtain better productivity. In China, the issue of availability or access to factors such as fertiliser, seed, and agrochemicals has been relatively covered; kudos to the Chinese efficient and effective production, dissemination systems and subsidies, farmers can easily purchase essential inputs. The constraints perceived by farmers in Hainan on the socioeconomic factors are majorly on the high cost of the factors. During the interview, Niger state farmersbitterly complained on the unavailability and high cost of fertiliser and other essential inputs (Figure 3(c)). One common cause of this as explained by some of the farmers is the "politicising of fertiliser", in local dialect referred to as "tahkinsiyasa".

The figures above compares the average rankings to each factor constraint by farmers in Niger state and Hainan province. The wider the ring the higher the constraints to rice productivity. The farmers in Niger state con- 


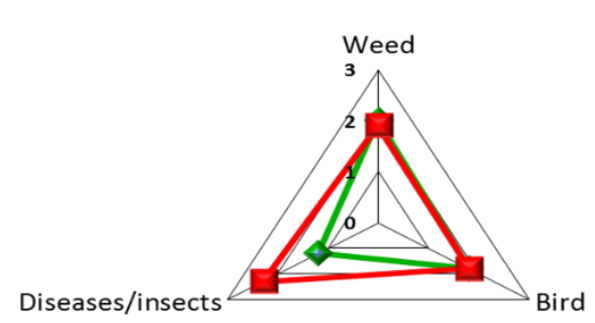

(a)

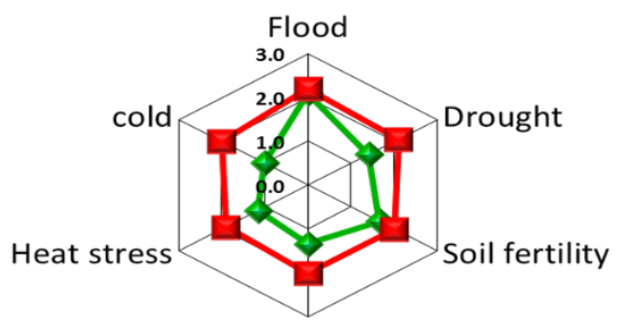

Soil erosion

(b)

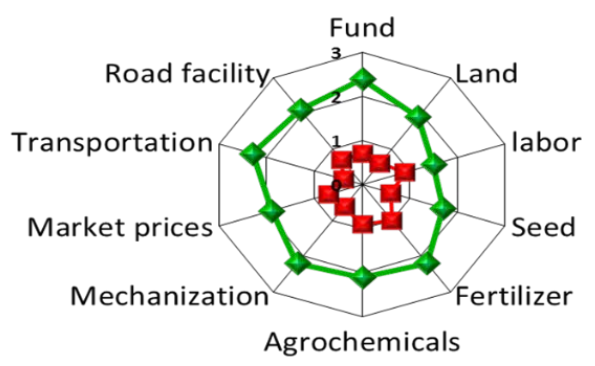

Niger state

Gainan

(c)

Figure 3. (a)-(c) Average ranking of constraints. (a) Biotic constraints; (b) Abiotic constraints; (c) Socioeconomic constraints.

sidered weed, flood, and all socioeconomic factors excluding labour and seed as the major constraints (on average ranked 2 and above) to rice production. Meanwhile farmers in Hainan province perceived all the factors abiotic constraints (on average ranked 2 and above) as the major constraints. The least ranked constraint was recorded on socioeconomic constraints by the Hainan farmers. In other words, the of efficient seed and fertiliser distribution, the continued government support in providing accessible roads, infrastructures and availability of credit facilities and other essential factors to the rural farmers helped improved rice yield. The survey reveals that the least ranked constraints of the farmers in Niger state are on the abiotic factors excluding flood. Reviewing the results of the survey on the socioeconomic perception of the farmers in Niger state towards rice production, many reasons were discovered as to the poor management of rice production. These setbacks cost not just the farmers but also the entire nation in terms of low efficiency and productivity in agriculture and reduced food security.

\subsection{Biplot Analysis}

\subsubsection{Constraints and Yield Level}

This research recognized there are significant perception gap among the different level of yield even within the same region. So the study exploits this by comparing the different yield levels between the two study areas.

The total average yield obtained by the farmers in Niger state and Hainan province are 2.7 and $4.1 \mathrm{MT} / \mathrm{ha}$ respectively. Yield varies across different regions and among the farmers. This section intend to identifying and estimating further the constraints among the different levels of each study area. Questions such as "are there any difference in the perception of farmers with better yield between the two regions?" or "does the low yield farmers experience similar fate between the two regions and at what magnitude?” would be answered.

The distribution of rice yield levels and yield gaps for the different yield groups in the two regions are presented in Table 1(a) and Table 1(b) below and then followed by the biplot diagram explaining the interaction of the production constraints with the yield groups of each study area. The yield group was calculated using Hainan yield data since the yield there is higher compare to Niger state.

The denoted yield group Y1/y1, Y2/y2 and Y3/y3 will be used interchangeably with low-yield, medium-yield and high-yield respectively for both regions. To determine the differences or similarities of the constraints among these yield groups, a multivariate biplot analysis was employed. As summarized by Kohler and Luniak (2005) [12], a biplot reveals the clusters of data, multicollinearity, and multivariate outliers of a dataset, and could also be used to guiding the interpretation of Principal Component Analysis (PCA). 
Table 1. (a) Descriptive statistics of yield group of Niger state farmers (MT/ha); (b) Descriptive statistics of yield groups of Hainan farmers (MT/ha).

(a)

\begin{tabular}{ccccccc}
\hline Yield group & Mean & farmers & Std. Dev. & Min & Max & Yield gap \\
\hline Y1 & 2.4 & $83 \%$ & 0.6 & 0.8 & 3.5 & 2.7 \\
Y2 & 4.0 & $13 \%$ & 0.3 & 3.5 & 4.7 & 1.1 \\
Y3 & 5.1 & $4 \%$ & 0.3 & 4.8 & 5.6 & -- \\
\hline
\end{tabular}

(b)

\begin{tabular}{ccccccc}
\hline Yield group & Mean & farmers & Std. Dev. & Min & Max & Yield gap \\
\hline y1 & 2.4 & 78 & 0.6 & 0.6 & 3.5 & 3.4 \\
y2 & 4.1 & 79 & 0.3 & 3.6 & 4.7 & 1.7 \\
y3 & 5.8 & 79 & 1.0 & 4.8 & 9.0 & -- \\
\hline
\end{tabular}

Source: Authors’ calculation from survey data 2013/2014.

The biplots below presents 3 observations (yield groups) and 19 variables (the constraints). Using Stata software: Command: biplot weed ...road facility, rowover (yield group. The explained variance of Niger state variables of the first component (dimension 1) in Figure 4(a) is about 68\% and the second component (about 32\%) giving a total explained variance of 1 i.e. 100\%, While Figure 4(b) shows that about $70 \%$ of the variance is explained by the first component and about 30\% explained for second component. The "rowover" distinguishes the yield groups by highlighting plot for each group identified by equal values of constraints.

In Figure 4, the arrows represents the constraints, the longer the length the higher the variance of the constraints among the yield groups and the angle between the lines approximates the correlation between constraints, and the distance between the yield groups approximates the Euclidean distance of any two of the group in the multivariate space of the diagram. From the biplot analysis, the constraints-flood, mechanisation, fertiliser and road facility depicts the highest variation among the yield groups, while the constraint cold depicts the lowest as seen from the length of its arrow. Given the closeness of the lines, there are a strong correlations between/among constraints-cold, agrochemicals and road facility; fertiliser and fund; pest and diseases, and transportation; soil erosion and mechanisation.

In the biplot, most of the constraints concentrates towards the right side where low and medium-yield is obtained. In another words, the farmers that had low and medium-yield perceive more the constraints to yield than the farmers with high-yield. Comparing the yield groups, farmers with high-yield majorly complained on the flood, high cost of labour and low market prices as the than other groups. Majority of the farmers in this group had access to fertiliser, better seed, and agrochemicals than the other two groups, this explains their high yield compared to their counterpart. The farmers with medium-yield on one hand majorly stressed on weed completion with the rice plant, high cost and/or availability of quality agrochemicals, poor road, limited access machine and soil erosion. While on the on the other hand, the farmers with low-yield complained more on inadequate fund to support the farm management processes, higher cost and unavailability of fertiliser, good seed, pest and diseases, drought, soil nutrient deficits and at-stress to rice plant. Most of the socioeconomic constraints lie between the low and medium-yield farmers especially on the low-yield groups.

The biplot (Figure 4(b)) illustrates that the constraints-drought, fertiliser, agrochemical, seed, birds and weed varies greatly among the yield group compared with other constraints. Compared to the biplot diagram of Figure 4(a), the perceive constraints of the different yield groups in Hainan shows low variance. From the above figure, the constraints with the lowest variance include market prices, labour and mechanisation. The correlation between the factor constraints on the other hand, is seen to be stronger among/between-pest, diseases, labour and fund; seed and transportation; fertiliser, agrochemical and cold.

In Figure 4(a), most of the socioeconomic constraints are located in the matric space of the low and high yield group. More of the abiotic constraints factors on the other hand reside near the medium and high-yield group. Comparative to the yield groups of the Hainan farmers, the disparity of the constraints among the relative yield level is minimal as shown in the biplot having many shorter lines. Nevertheless, some differences could be 


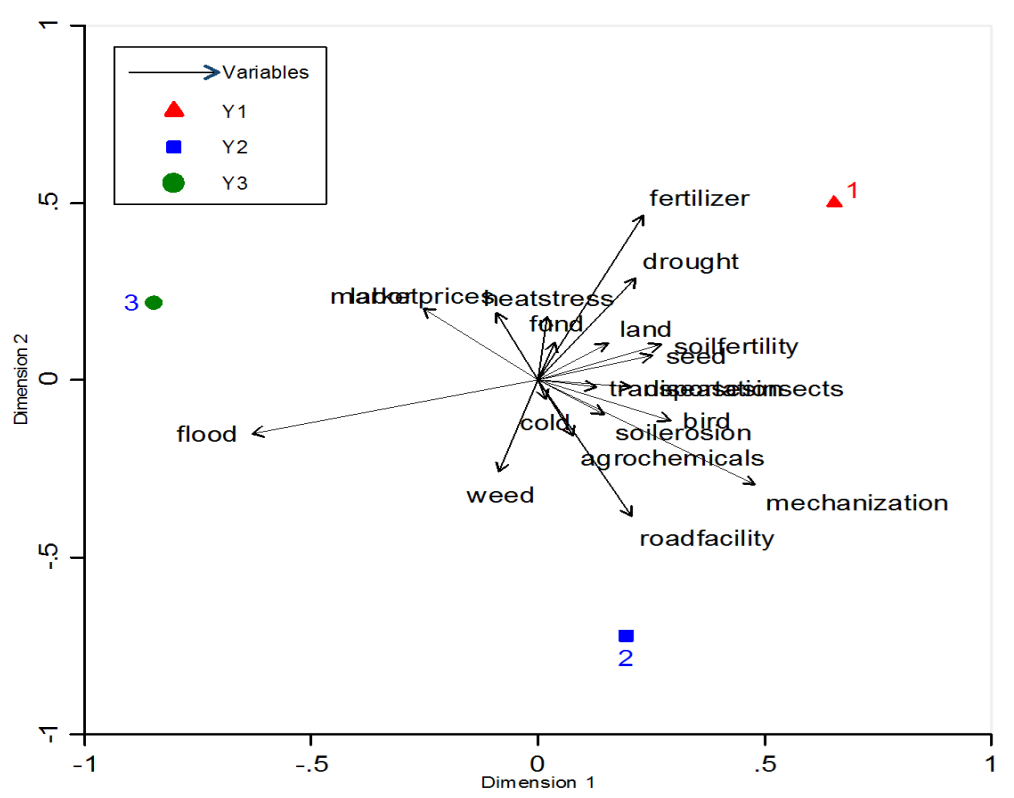

(a)

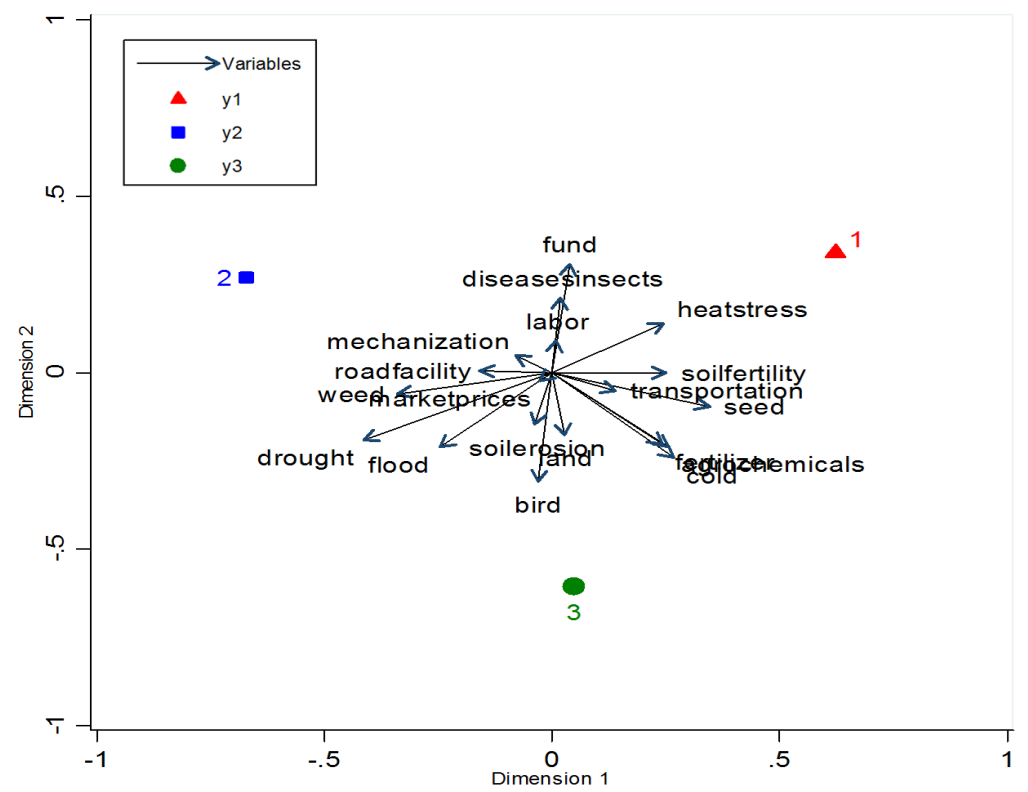

(b)

Figure 4. (a) biplot of rice yield group and constraints to yield (Niger state); (b) biplot of rice yield group and constraints to yield (Hainan province).

seen. The farmers with high-yield dominantly perceive constraint-bird, flood, soil fertility, soil erosion, price of fertiliser and agrochemicals higher than the other two groups. This explains the increase use of fertiliser to increase the nutrient for the rice plant since their perception on the constraints of soil nutrient to better yield is high. With relatively adequate fund and availability of essential input that enhances yield, the higher the farmer perceive the threat of a constraint factor the more the use of these essential inputs regardless of the standard application. In the case of Niger state farmers, unavailability and/or high cost been the foremost problem, thus even though the farmer ranked constraint high, the essential inputs to combat the constraints are scarce or inadequate. The medium-yield group compared to other groups, majorly complained on constraint-weed, drought, cost of seed and farm mechanisation, and lastly the low-yield group stressed on constraint-soil fertility, fund, heat stress on rice plant cost of fertiliser and machine. 
Furthermore, the socioeconomic constraints which are ranked highest in Niger state are perceived more by the low-yield farmers. On the contrary, the high-yield group in Hainan perceived more than the other groups. With the availability of essential inputs to enhancing yield, the higher the perception of the constraint to yield the more the farmers apply the input to reduce the impact of the constraints but on another hand, if there is scarcity of these yield enhancing inputs then the above statement would not hold as in the case of some farmers in Niger state. Moreover, the result of the biplot analysis for the two studied areas clearly indicated that there are opportunities for yield improvement and reduction of yield gap by making the essential input available to the farmers more specifically in Niger state.

\section{Conclusions and Recommendation}

This study measures and analyzed the perception of rice farmers towards three categorized production constraints (namely biotic, abiotic and socioeconomic constraints) of Niger state of Nigeria and Hainan of China. Rice yield of both region was divided into 3 groups (low-yield, medium-yield and high-yield). Biplot analysis was then used to analyse the interaction between the yield groups of regions and the production constraints experienced by the farmers.

The average yield obtained by the farmers in Niger state and Hainan province are 2.7 and $4.1 \mathrm{MT} / \mathrm{ha}$ respectively ceteris paribus, rice productivity in Hainan out ways that of Niger state. The study comparatively shows the vivid differences in the abiotic and socioeconomic constraints between the two regions. While the socioeconomic constraint is higher in Niger state, the abiotic constraint record higher in Hainan province. In Niger state, there are larger variations between the low and higher yield farmer than that of Hainan farmers were the variation is minimal. The variations in Niger state are majorly seen in the socioeconomic constraint factors. The Biplot analysis explains that the perceive constraints of the different yield group in Hainan shows low variance while that of Niger state shows higher variance among the three yield groups. In Hainan province, the high and medium-yield group farmers ranked more constraints greater than the farmers with low-yield although with little variance. While in Niger state, the low and medium-yield groups ranked more constraint to yield higher than the farmers with high-yield. The policy implication of the findings in this study indicates that great potential remain to further improve rice yield in both regions. The evidence from the rice farming system in Hainan shows that yield in Nigeria could be substantially higher when the appropriate investment on essential inputs given that the major factor constraint which Nigeria faces (socioeconomic constraints) can be control with the right investment in the sector.

\section{Acknowledgements}

This paper was supported by the CAAS Science and Technology Innovation Project (number: CAAS-ASTIP2016-AII), founded by Chinese Academy of Agricultural Sciences and Technology.

\section{References}

[1] GRiSP (2003) Quality Seed Production in Hybrid Rice. Global Rice Science Partnership (GRiSP) Rice Almanac 2013, 52-53, 83-85; Sindhu and Kuma. http://books.irri.org/9789712203008_content.pdf

[2] USITC (2015) Rice: Global Competitiveness of the U.S. Industry. United States International Trade Commission (USITC). https://www.usitc.gov/publications/332/pub4530.pdf

[3] Eponou, T. (1993) Partners in Agricultural Technology: Linking Research and Technology Transfer to Serve Farmers. ISNAR Research Report No. 1, International Service for National Agricultural Research, The Hague.

[4] Erenstein, O. and Lancon, F. (2003) The Nigerian Rice Economy in a Competitive World. Constraints, Opportunities and Strategic Choices. Report of the Final Technical Workshop Held in IITA on August 20-21, 2003.

[5] NBS (2013) Federal Republic of Nigeria. http://nigeria.prognoz.com/en/Map/?7Dt5TDYdnkG4wfwXzAPnSg

[6] Atteh, O.D. (1984) Nigerian Farmers' Perception on Pest and Pesticidesll. Department of Geography, University of Ilorin, Ilorin, Nigeria. Insect Sri. Applic., 5, 213-220.

[7] Emechebe, A.M., Ellis-Jones, J., Schulz, S., Chikoye, D., Douthwaite, B., Kureh, I., Tarawali, G., Hussaini, M.A., Kormawa, P. and Sanni, A. (2004) Farmers' Perception of the Striga Problem and its Control in Northern Nigeria. Expl Agric, 40, 215-232. http://dx.doi.org/10.1017/S0014479703001601

[8] Escalada, M., Wang, L., Yuan, Q.H., Cai, D.C. and Heong, K.L. (2012) Baseline Survey Report. IRRI, Conserving 
Arthropod Biodiversity and Ecosystem Services in Rice Environments of Hainan Island. http://hainanproject.org/wp-content/uploads/2012/01/Hainan-baseline-survey-report-jan-2-2012.pdf

[9] Aerni, P. and Yu, W. (2010) Empirical Evidence of Stakeholders' Perception on Sustainable Agriculture in China. Proceedings of 2010 Chinese Agricultural Economic Review (CAER), International Food Policy Research Institute (IFPRI) International Annual Conference on Agriculture and Wealth of Nations, 16-17 October 2010, Beijing.

[10] Alarima, C.I., Adamu, C.O., Masunaga, T. and Wakatsuki, T. (2011) Constraints to Sawah Rice Production System in Nigeria. J Hum Ecol, 36, 121-130.

[11] (1996) Gower and Hand.

[12] Ulrich, K. and Magadalena, L. (2005) Data Inspection Using Biplot. The Stata Journal, 5, 208-223

[13] Johnson, R.A. and Wichern, D.W. (2007) Applied Multivariate Statistical Analysis. Sixth Edition, Pearson and Education.

[14] Atteh, O.D. (1984) Nigerian Farmers' Perception on Pest and Pesticides. Department of Geography, University of Ilorin, Ilorin. Insect Sri. Applic., 5, 213-220.

[15] Sinzogan, A.A.C., Van Huis, A., Kossou, D.K., Jiggins, J. and Vodouhè, S. (2004) Farmers’ Knowledge and Perception of Cotton Pests and Pest Control Practices in Benin: Results of a Diagnostic Study. NJAS. http://dx.doi.org/10.1016/s1573-5214(04)80018-6

[16] Ojehomon, V.E.T., Adebayo, S.B., Ogundele, O.O., Okoruwa, V.O., Ajayi, O., Diagne, A. and Ogunlana, O. (2009) Rice Data Systems In Nigeria (National Rice Survey 2009). Building a Rice Data System for Sub-Saharan Africa. Africa Rice, National Bureau of Statistics, National Cereal Research Institute, Nigeria Institute for Social and Economic Research (NISER), Department of Agric. Economics, University of Ibadan.

[17] Atera, E.A., Itoh, K., Azuma, T. and Ishii, T. (2012) Farmers’ Perception and Constraints to the Adoption of Weed Control Options: The Case of Strigaasiatica in Malawi. Journal of Agricultural Science, 4. http://dx.doi.org/10.5539/jas.v4n5p41

[18] Daniel, L. (2003) Crop Farming in China: Technology, Markets, Institutions and the Use of Pesticides. Shaker Verlag Aachen, Institutional Changes in Agriculture and Natural Resources, 17. 